\title{
ALCOHOL CONSUMPTION IN ELDERLY PEOPLE ACROSS EUROPEAN COUNTRIES: RESULTS FROM THE FOOD IN LATER LIFE PROJECT
}

\author{
Maria Daniel VAZ De Almeida ${ }^{1}$, KATe DaVIDSON ${ }^{2}$, \\ Cecilia De Morais ${ }^{1}$, Helen Marshall ${ }^{2}$, Silvia BofiLl ${ }^{3}$, \\ KLAUS G. GRUNERT ${ }^{4}$, KATARZYNA KOZLOWSKA ${ }^{5}$, \\ YOLANDA LACASTA ${ }^{3}$, SimONA MARTINES ${ }^{6}$, \\ YlVA MATTSSON-SYDNER ${ }^{7}$, HeNRIETTE BOEL NIELSEN ${ }^{4}$, \\ GUDRUN SELTMANN $^{8}$, ANNA SZCZECINSKA ${ }^{5}$, MONIQUE RAATS ${ }^{2}$, \\ MARGARET LUMBERS ${ }^{2}$ AND THE FOOD IN LATER \\ Life Project TEAM
}

\begin{abstract}
The aim of this paper is to identify social and cultural aspects of alcohol consumption in a sample of older people living in their own homes, in eight different European countries. We explore several aspects of alcohol consumption, establishing comparisons between genders, age groups and living circumstances. The phenomenon of alcohol consumption within these countries and cultures is compared in order to gain a better understanding of similarities and differences.
\end{abstract}

In general, research on alcohol consumption is problem-oriented and only a minority of the drinking population is studied. Most current literature emanates from the discipline of medicine and is principally concerned with biochemical interactions with organs (including the brain) that are detrimental to good health. In consequence, the influence of lifecourse and social circumstances has been somewhat lost in the research process. The phenomenon of 'normal drinking' and different cultural drinking patterns are even less frequently explored issues. Countries that have a so-called 'Temperance tradi-

Ageing International, Fall 2005, Vol. 30, No. 4, pp. 377-395. 
tion' (also called 'Nordic' or 'ambivalent' drinking cultures) and ambivalent attitudes to alcohol have been responsible for most research on this field, while cultures in which drinking is not seen as a problem ('non-Temperance', 'Mediterranean' or 'integrated' drinking-cultures) have been less researched (SIRC, 1998).

Alcohol consumption in the perspective of the social and behavioral outcomes has to be explained by other factors than ethanol effects only, being influenced mainly by the people's culture and social influences. There is an enormous cross-cultural variation in the perception of alcohol related behavior. In some societies (such as the UK, Scandinavia, US and Australia), especially among younger cohorts, alcohol is often associated with violent and anti-social behavior. Whilst in others (such as Mediterranean cultures), drinking is more likely to be associated with a peaceful and harmonious behavior. These variations can be attributed not only to different levels of alcohol consumption, but to cultural beliefs about alcohol and expectations regarding the effects of alcohol and to the social norms regarding drunkenness.

In general, societies with positive beliefs and expectations about alcohol (Mediterranean countries) have significantly fewer alcohol-related problems; the opposite situation is true in countries (such as Nordic ones) that have negative or inconsistent beliefs and expectations, and have higher levels of alcohol-related problems. The analysis across different cultures also suggests that there are four items considered 'near-universal constants': proscription of solitary drinking, prescription of sociability, social control of consumption and behavior, and restrictions on female and 'underage' drinking (SIRC, 1998).

\section{Alcohol Consumption across European Countries}

The latest World Health Organization (WHO) global report on alcohol (2004) categorizes alcoholic beverages as beer, wine or spirits. The report provides an analysis of the top 20 countries with the highest consumption for each category, using the recorded adult per capita (APC) in liters of pure alcohol for the three specific beverages. Different drinking patterns are quite evident. For the European countries, beer has a higher consumption in countries such as the Czech Republic, Ireland, Germany, Denmark and the UK. Wine is consumed in higher quantity in France, Portugal, Italy Spain, Denmark and Germany (southern). Regarding spirits consumption, only a few European countries were found in the list of 20 top consumption countries, namely the Republic of Moldova, the Russian Federation, Latvia and Bosnia and Herzegovina. The report identifies these differences, linking them to wine production across the countries (WHO, 2004). The classification is based on wine production and consumption:

- high level of production and a high level, but decreasing level of consumption: France, Greece, Italy, Portugal and Spain; 
- medium level of production, moderate level of consumption and a stable or slightly increasing consumption: Austria, the Czech Republic, Germany and Switzerland;

- non-production countries that have experienced a strong increase in wine consumption: Belgium, Ireland, the Netherlands, Nordic countries and the UK (WHO, 2004).

There has been considerable attention to alcohol consumption and harm across European countries (IAS, 2005). However, reports have tended to focus on younger adults, particularly those who indulge in underage drinking and those who suffer from pathological conditions resulting from harmful consumption. Less attention has been paid to older drinkers (O'Connell et al., 2003). Although most older people do not drink at levels associated with a 'drink problem', their use of alcohol is nevertheless 'more widespread and harmful than is realized outside some specialists in the health and social care professions' (Clough et al., 2004:1). Ageing tends to reduce the body's tolerance of alcohol and to amplify risk factors. Due to a decrease in total body water, a lower alcohol intake is needed to cause a given concentration in the blood, as well as intoxication and addiction (Vestal et al., 1977; Hepple, 1996; Chin et al., 2004). However, there is some evidence that low levels of drinking, especially of red wine, can have beneficial effects on health and wellbeing (Thun et al., 1997; Truelsen et al., 2002; Djousse et al., 2002). It is not the intention of this paper to identify 'problem drinking' in a sample of European older people. Rather, it examines older people's perceptions of alcohol consumption as a social act.

\section{Methods}

Food in Later Life is an European project carried out with financial support of the European Communities, under the 5th Framework Program for Research, Technology and Demonstration, specific RTD program Quality of Life and Management of Living Resources, Key Action 1 'Food, Nutrition and Health' (www.foodinlaterlife.org). Eight European countries were involved with the project: Denmark (DK), Germany (G), Italy (I), Poland (PL), Portugal (PT), Spain (SP), Sweden (SE) and the United Kingdom (UK). A variety of recording instruments were used, including a Background Questionnaire, seven-day food diaries (shopping and consumption) and in-depth semi-structured interviews lasting approximately an hour.

Such a large, multi-cultural, multi-disciplinary and multi-method project is not a new development in academic research. However, our project is exceptional inasmuch as all the partners carried out simultaneous data collection using all the methods described above. This permitted comparative analyses of contemporaneous data and thus has contributed a rich and unique body of research findings from western, northern, southern and central Europe. This 
pioneering approach is reflected in the list of authors who have contributed to the article. Some of the methodological issues are addressed in the discussion section here, but we consider they warrant a paper in their own right-such were the joys and challenges experienced during the project.

This article reports on 644 in-depth, semi-structured interviews collected by the eight European partners from independently-living seniors, that is, people not living in residential or nursing homes. In 2004, each country conducted interviews (Table 1), according to quotas for genders (men/women), age groups (ages 65-74 and 75 or older) and living circumstances (living alone and living with others). All the data collection and subsequent analyses were carried out in the language of the country and the principal findings translated into English, the common language of the project. The data from interviews were transcribed and analyzed using MAXqda (2001).

The matrix is complex and the data collected from the quotas (age, gender and living circumstances) are highly relevant for individual country analysis, or cross-cultural analysis of discrete categories. However, for this paper, we have taken a broad spectrum cross-cultural approach that defines the participants by gender and country. Therefore, the paper reports the narratives of 40 males and 40 females from DK, G, I, PL, and PT, 39 males and 40 females from SE, 40 males and 43 females from SP and 41 males and 41 females from the UK. For the quotations, the respondents are identified by gender and country, for example, $\mathrm{M}=$ Male, $\mathrm{PT}=$ Portugal: $(\mathrm{M}, \mathrm{PT}) ; \mathrm{F}=$ Female, $\mathrm{G}=$ Germany: $(\mathrm{F}, \mathrm{G})$.

The semi-structured schedules sought to investigate older people's eating behaviours: what they consumed and with whom they ate. We were interested in how they expressed what constituted a 'proper' or 'ideal' meal and what social interaction, if any, contributed to their notion of a 'proper' or 'ideal' meal. We were particularly interested in how their food consumption and re-

Table 1

\begin{tabular}{|c|c|c|c|c|c|c|c|c|c|}
\hline \multicolumn{10}{|c|}{ Sample distribution across countries } \\
\hline \multirow[b]{3}{*}{ COUNTRY } & \multicolumn{4}{|c|}{ MEN } & \multicolumn{4}{|c|}{ WOMEN } & \multirow{3}{*}{$\begin{array}{c}\text { TOTAI } \\
\mathbf{n}=644\end{array}$} \\
\hline & \multicolumn{2}{|c|}{ 65-74 years } & \multicolumn{2}{|c|}{$\geq 75$ years } & \multicolumn{2}{|c|}{ 65-74 years } & \multicolumn{2}{|c|}{$\geq 75$ years } & \\
\hline & LA & LWO & LA & LWO & LA & LWO & LA & LWO & \\
\hline Denmark (DK) & 10 & 10 & 10 & 10 & 11 & 10 & 9 & 10 & 80 \\
\hline Germany (G) & 10 & 10 & 10 & 10 & 11 & 10 & 9 & 10 & 80 \\
\hline Italy (I) & 10 & 10 & 10 & 10 & 12 & 9 & 10 & 9 & 80 \\
\hline Poland (PL) & 10 & 10 & 10 & 10 & 11 & 9 & 10 & 10 & 80 \\
\hline Portugal (PT) & 10 & 10 & 10 & 10 & 10 & 10 & 10 & 10 & 80 \\
\hline Sweden (SE) & 9 & 10 & 10 & 10 & 12 & 11 & 10 & 7 & 79 \\
\hline Spain (SP) & 10 & 7 & 10 & 13 & 9 & 10 & 11 & 13 & 83 \\
\hline $\begin{array}{l}\text { United Kingdom } \\
\text { (UK) }\end{array}$ & 11 & 10 & 8 & 12 & 9 & 12 & 10 & 10 & 82 \\
\hline
\end{tabular}

LA = Living Alone

LWA = Living With Others 
lated social interaction had changed over their life course, and how this might be different for men and women. We did not ask specifically about alcohol consumption (number of units, types of alcohol), but the analysis in all eight countries revealed that virtually all the respondents talked spontaneously about alcohol as it related to their perception of, and adjunct to food consumption and commensality. Once the analysis from the partners was collated, we noted that there were considerable gender, and importantly, cultural differences in the narratives about the status of alcohol in their everyday lives.

Our approach to the analysis of the rich qualitative data drew heavily on a grounded theory perspective (Strauss and Corbin, 1990) that permits the percolation of pertinent themes through the data. We were sensitive to what the data were 'telling' us (Silverman, 1993), but worried that we were imposing our own pre-conceived notions of cultural differences, as expressed in our introduction (Nordic vs. Mediterranean). However, Lofland and Lofland (1995) reassure us:

You get from the data, topics, and questions, on the one side, to answers or propositions on the other, through intensive immersion in the data, allowing your data to interact with your intuition and sensibilities as these latter are informed by your knowledge of topics and questions (Lofland and Lofland, 1995: 184 original emphasis).

We therefore felt confident that we were breaking new ground in terms of research on cross-cultural attitudes to alcohol consumption in older men and women.

\section{Results}

This paper is organized in three main sections in which we compare the attitudes of older people to alcohol consumption among the participants from all the eight countries. We were thus able to obtain information from several aspects and compare alcohol consumption in countries from the Mediterranean and northern Europe. The sections are: 'Alcohol consumption narratives of older people', 'Gender differences in the narratives' and 'Specific cultural differences between the eight countries'.

\section{Alcohol Consumption Narratives of Older People}

We found several attitudes toward alcohol consumption across the eight countries. In general, the narratives may be divided between those who drink, and do so regularly; those who drink occasionally; and those who do not drink or hardly ever drink.

Firstly, we examine the attitudes of those who consume alcoholic beverages as a daily routine. Across all countries, respondents, who reported drink- 
ing regularly, cited enjoyment as the central motivation to drinking. Some respondents in Germany mentioned that a glass of good wine upgrades a simple meal. The enjoyment of drinking a glass of wine or beer is more frequent when they meet together with their friends or family. In Italy, the alcohol consumption was not only associated to meals but also to being in company of others, as a matter of a glass of good wine being part of a meal shared with others: 'When I am together with people ... I must eat, I must drink' (M, IT).

Alcohol consumption is connected with culture and has also social and psychological implications, as we found in some narratives. For some older people in Germany, for example, the glass of wine or beer is 'a nice treat for yourself', in some cases it is a substitute for sweets or desserts (for some diabetics). Alcoholic beverages were considered important within domestic and routine environments and felt to mark aspects of routine for Polish participants, making them more enjoyable/relaxing such as the daily main meal, the weekend, the end of the day or even their daily life.

Alcoholic beverages are so rooted in some cultures that for these people, drinking a glass of wine or another alcoholic beverage is considered as being part of the meal: 'Now, lunchtime I have a glass of wine when I have my meal because I enjoy that' (F, UK).

In some countries, it is indeed unusual to talk about 'alcohol' when referring to wine or beer. Interestingly, the older people who drank, tended to name the beverage, for example beer, wine, liqueur or schnapps (PT, SP and $\mathrm{G})$ and only those who were abstainers used the generic term 'alcohol' or 'alcoholic drink'.

And in the evening, my husband sits down in the living room ... to watch some TV ... with his block of chocolate... And I go to the living room with my quarter liter of white wine $(F, G)$.

Many participants discussed the appropriateness of particular drinks to particular foods. This was especially true for wine consumption, as many informants talked about drinking white wine with fish dishes, and red wine with meat dishes (PT, SE); in Sweden, in some cases this culinary rule is related to beer.

If you have a dish of meat you may have beer or wine to drink (M, SE).

I grill some sardines and then I drink a little wine, only a small glass (F, PT).

Some Portuguese women emphasized the fact that they would drink a glass of wine whenever they eat fish. This was also a key aspect for many respondents in the UK, who felt alcoholic beverages was integral to 'proper' meals. In Denmark, the group that drinks every day is older people who drink a glass 
of wine whilst dining. The narratives show that regional or seasonal customs have an influence on what is drunk when and that these customs are cherished and often involve some kind of social interaction, e.g., onion pie and new wine in the autumn, champagne for the New Year, white wine in asparagus season. Respondents in Germany mentioned that they drink certain alcoholic beverages with certain meals, e.g., wine only with savory dishes not with sweet dishes.

In all countries, especially those considered 'Mediterranean' such as Italy, Portugal and Spain, the most frequently named drink was wine, followed by beer. In the North European countries, we found more references to spirits. In Poland, they emphasized that alcohol (especially vodka) is an integral part of some typical Polish dishes; in their opinion it is necessary to drink a glass of vodka with a Polish dish made of sauerkraut, sausage and mushrooms, to pork chops, tripe or herrings. '[B]igos (tripe): a glass of vodka is just obligatory to eat this food properly' (M, PL).

Alcoholic consumption was much more evident, for all countries, when we asked about the concept of an 'ideal meal'. The narratives show that for the majority of respondents, drinking a glass of wine or beer is an essential part of any meal and when they have guests (family, friends) for meal events, and also a more common routine for meals at weekends. Several respondents also mentioned other types of alcoholic beverages, besides wine and beer, as appetizers or beverages that are related to celebration, mostly champagne and whisky or liquors. When describing an ideal meal a lot of the informants say that they also want 'something to drink'.

Many of the women, who do not drink routinely, mention they enjoy a glass of wine or champagne for the special occasion.

In the New Year ... then I drink a glass of champagne or Porto wine. On those occasions I drink a small glass of wine... (F, PT).

When Diego (son) comes some weekends we open a bottle of 'cava'. I like it very much and that way I can drink it because I don't do it alone (F, SP).

Going out or eating out, in the understanding of the German subjects, seems to be linked to drinking alcohol. Therefore respondents who do not drink alcohol remark that they tend not go out to pubs or taverns. This implies that people, who cease or have been advised to cease consuming alcoholic beverages for health reasons, lose part of their social activities, especially if they now live alone. Furthermore, the narratives show that respondents do not enjoy going out by themselves and hardly anyone does so, certainly not women. Drinking and eating alone have different social connotation on the different countries and depends also of their living circumstances and gender, as will be developed later. 
Lunch and dinner were the meal events when the consumption of an alcoholic beverage was most reported in all countries. In most countries, alcohol was not taken with a light meal or snack. Nevertheless, in Portugal and Spain, we found that men in particular reported having a drink with light snacks in the afternoon.

I drink half a glass of beer or wine at the meals (F, IT).

When I have the lunch ready, I take a 'vermú'. ... Sometimes a glass of wine or a beer, it depends, before lunch (M, SP).

I am associated to 'Figueirense' (football club).... And sometimes I go there, spend some time playing cards. They prepare some snacks... I always eat a sandwich, usually with fried fish ... and I drink a glass of wine. Then I go back home to have dinner' (M, PT).

In Germany also, older people have wine or beer in the evening, whether they were having a warm or cold dinner. For many, having a glass of wine is a nice way to end the daily routine.

Seasonal variation in alcohol consumption was found in other countries, but in these circumstances it was not related to the traditional foods but to thirst and the need to drink beverages that are more refreshing. In Spain, older people reported a greater need to drink alcohol in some seasons, although it should 'never be in excess'. They considered that water in the summer does not 'kill the thirst' and beer is more efficient in these occasions because it 'cools the body'. Some Portuguese older people also mentioned that beer tastes better in the summer and it is a good drink when they are thirsty.

A particularity was that the cold weather in the North European countries was a good reason for having a drink. This was narrated by some older people from Poland and Germany, for example:

I've always enjoyed drinking ... but it's not my favorite activity, it's not so that I have to drink, etc. When someone feels cold he can have a glass of alcohol, it wouldn't do any harm (M, PL).

In winter, for example, as I already mentioned, I like to eat game, if there is good game and in the Southern Germany way with 'Spätzle' noodles or something like that, some kind of snack before, such as little bit of salmon or something else, something special, that is, and then in a pleasant atmosphere, and a nice glass of wine is not to be sneezed at either $(\mathrm{M}, \mathrm{G})$.

The narratives in some countries, especially those from the north, showed that some older people feel the need to justify their alcohol consumption, as if it was seen as socially unacceptable. Some Swedish older people talked about 
how they have a glass in the middle of the week and then they specifically point it out as something 'odd'. Some tried to excuse their behavior talking about their age and that they believe that they now are so old that a glass of wine every day must be fine. This social acceptability becomes more relevant in the women's narratives, but was also found in men's: 'I may have a beer sometimes ... well that is not proper on week days maybe...' (M, SE).

\section{Reasons for Not Drinking Alcohol}

Respondents who said they were occasional drinkers or those that said they did not drink at all explained this fact with several reasons, such as taste, health, cost or 'bad' aspects to drinking (drunkenness, alcoholism). In some countries, one of the more important reasons for not drinking alcohol was because of poor health. In the Portuguese narratives we find good illustrations, when they mentioned some chronic diseases, such as Type 2 diabetes or psychological or neurological disease such as emotional problems or, in one case, epilepsy. Interestingly, health aspects and abstinence from alcohol were rarely mentioned among the Polish or Swedish samples who tended to mention the need of being more careful with alcohol if they suffered from chronic diseases or when they were afraid of developing some illnesses.

More important for the countries of northern Europe (SE, UK) was the danger of drinking and driving, which reflects their concern with their strict laws. They feel it is not possible to do both, and being at a dinner party or at a restaurant is often associated to not being able to drive back home on their own car. 'Well, sometimes you take a taxi back home and pick the car up the day after' (M, SE).

\section{Alcohol Consumption as an Aid to Health}

In countries such as Denmark, Poland, Germany, Spain and Portugal, we found narratives from participants that explained that they consume alcohol for health reasons. Alcohol, in Poland, was considered very good for stomach or digestive problems or when one was feeling cold; and vodka was considered to have an healthy impact on the body. In Germany, several respondents mention that they have 'schnapps' after a heavy meal to help digestion. Schnapps is considered the perfect end to a good meal, usually with several courses.

It turned out that the alcohol is very good for digestion (M, PL).

About my drinking habits, I can say that as well, yes. I drink two three times a week ... wine. But of course wine contains no sugar, right? I mean dry, absolutely dry. And the strange thing is, that my sugar levels sink after I drank wine. So that I can even eat 
more if I want to. Myself ... it is actually the case, yes that the wine reduces the sugar, yes ... experience $(\mathrm{M}, \mathrm{G})$.

Drinking a glass of wine or whisky was often justified by medical recommendations in countries such as Portugal and Spain. Because the doctor told it was 'good for the heart', 'good for the bones' or 'good for blood', for example: 'My doctor even said to me that I should drink a glass of wine during the meal because it was good for me, as I have a very "poor" blood' (F, PT).

Some women in the UK also narrated the different uses they had for alcohol, usually either as a 'pick me up' when they were tired or as an aid to help them sleep. Interestingly, these functions/uses of alcohol were not only concentrated in the narratives of women respondents, but specifically in the narratives of the older women living alone. In fact, it is reported (IAS, 1999) that people may use alcohol to help them sleep, especially if they are experiencing some sort of physical or emotional distress.

\section{Gender Differences in Narratives of Alcohol Consumption}

We examined gender differences among our population due to the great evidence of such disparities in alcohol consumption between older men and women (Wilsnack and Wilsnack, 2002). The most relevant are cultural influences, stigmatization of women's drinking and effects of changing gender roles. All the participating countries found some gender differences in alcohol consumption. The most evident was that men tended to talk about alcohol more spontaneously and frequently than women. In Italy, for example women tended not to talk a lot about alcohol; in fact, most of them did not mention their consumption at all during the interview, though they often use alcohol as an ingredient in many recipes.

\section{Similarities between genders}

For both men and women, wine was the most preferred beverage in all countries. In general, one or two glasses of wine are consumed during the meals. In Italy, some of the men drink wine regularly during the meals and the consumption is strictly associated to the company of other people and to large meals; others said their consumption was very limited or, only on special occasions.

Another similarity between genders was found in the Spanish narratives, where alcohol was highly linked to commensality and sociability for both men and women, and also that both groups talked about the use of alcohol when cooking. These older people found very useful the use of wine or other alcoholic beverages to give food 'a good taste': 'I like to cook, when you prepare rabbit for example.... You put a glass of cognac and when it's all cooked you can add water or beer' (F, SP). 


\section{Most Marked Differences}

When women reported abstaining from alcohol they offered several reasons, but especially non-enjoyment of the taste, or because they have never, or rarely taken alcohol. Women who had been advised by health professionals to give up, or drastically reduce alcohol consumption were less likely than men to find it problematic; dissatisfaction with the fact of not being able to drink due to health constraints was more evident within the men's narratives.

When women did talk about alcohol, there were some differences across countries in how they expressed their attitude, especially regarding the purposes and circumstances for alcohol drinking. Usually, among female participants, the consumption of alcohol was more associated with main meals or special occasion or when they meet friends and relatives in most countries (UK, PT, SP, DE).

In Spain, although women tend to talk less about alcoholic beverage consumption than men, the ones who do it talk, do it in a very positive way, referring 'liking beer' or the pleasure of drinking a 'vermú' when they are having a snack at a bar. The UK women were more likely to emphasize drinking alcohol as a source of enjoyment than the men who were more likely to talked about consumption as 'routine'. Many women attached great importance to having alcohol when entertaining and felt this was integral to sharing a special meal: 'Weekends, we usually have a bottle of wine.... It just sort of goes with the meal on a weekend for some reason, you know' (F, UK).

In Sweden, it was interesting that no woman reported drinking schnapps, but some men did. It was also more common that Swedish men mentioned beer when talking about alcohol beverages whereas Swedish women most commonly reported that they were more likely to drink wine than any other alcoholic beverage. In the northern countries (PL, G) women tend to drink small quantities of wine usually in social occasions, when they meet with friends in their homes and prepare a sweet snack, tea or coffee. Portuguese women talked about drinking a glass of wine during meals in their daily routine but especially when they are accompanied and associated to certain foods, as mentioned before.

For the UK women, such was the enjoyment that a drink added to a meal it was generally regarded as acceptable to drink alone with a meal, as long as it was in moderation.

It [wine] adds to the enjoyment of a meal, but obviously not too much, which I don't—at the moment. No, it is a bad habit to drink too much on your own anyway. I know that it is easy to get into the habit of having two (glasses) but I don't. Anyway, I don't enjoy more than two, so I don't' (F, UK).

One important point is that unacceptability of alcohol consumption was much more implicit in the women's narratives in some countries when com- 
paring to men's. In Germany, only women discussed alcohol consumption as conflicting with social norms, that is, drinking alcohol during the day is considered forbidden and some expressed guilt for even feeling like doing so. Or they mention that they enjoy having a glass of wine and then emphasize that they are not alcoholics. Danish women were also more concerned than men that their consumption of alcohol is not seen as an addiction to drinking. In the Portuguese interviews, we found the need to stress that even wine consumption during meals was very small. It was marked by expressions such as 'small glass', 'few drops' or 'I only taste it'. Most of the occasional consumers stressed the fact that they cannot drink a lot of alcoholic beverages because it interferes with their physical and psychological states and do not want to feel embarrassed with more inappropriate behaviors for a woman (association with drunkenness and alcoholism).

In some interviews, we could identify the need of affirming that they did not consume any, or very little alcoholic beverage, remarking they considered it unacceptable for women.

And then I fix myself some rice or, well and then a nice wine with it, I mean it is not that nice, usually rosé wine or Weißherbst (a special kind of German rosé) or something like that, a small glass, it's not like I am a big alcoholic, no alcohol drinker' (F, $\mathrm{G})$.

In northern Europe, the narratives regarding alcohol consumption from men involved more reference to spirits and were usually associated with male social events, such as watching football games on TV with other men. Many Polish men would regularly drink vodka. They usually organized typical male parties, associated with watching TV (football games) or playing cards and prepared special meals like herring and tripe. Male sociability nearly always incorporated some alcohol.

Interestingly, in the UK, many men focused on the control they had over drinking, often defending their drinking habits in relation to health benefits and other health behaviors. Those who considered themselves as heavy spirit drinkers tended to justify their actions in that they liked drinking and that they were in command of their drinking and could stop when they wished to. However, in southern Europe, fermented alcohol (wine and beer) was still more likely to be drunk at male gatherings. In Portugal, some men said they enjoyed drinking a glass of wine or beer when they meet with friends at taverns or at social clubs, while they chatted or played cards. The Spanish men narratives emphasized the consumption of alcoholic beverages at some meals, and for them it was not just a matter of consuming alcohol but it is part of the meal itself, including the daily consumption of wine at lunch and sometimes a beer during the late morning snack (before lunch).

In some countries (PT, DE), it was mostly men that associated their daily wine consumption with health benefits and the medical recommendations they 
had from the family doctor. This justification was usually enhanced by some verbal expressions that suggest a small to moderate consumption, such as 'small glass of wine', 'half glass' or 'a little drop'. One particularity was that some men also talked about mixing water with wine or soda, emphasizing that the alcohol consumption is very low in spite of being an everyday routine. Italian, German, Danish and Portuguese men talked more frequently of alcohol consumption in their daily routine than women and appreciate drinking a quality wine.

The perfect meal, that is to be together with your family and good friends around the table. And to get some good wine (M, DK).

A good wine sets off a meal ... for an ideal meal a good bottle of wine is fundamental $(\mathrm{M}, \mathrm{IT})$.

In all the countries, exclusive male sociability was almost always lubricated by alcohol and considered essential to the commensality experienced within the groups: 'I was a member of the Polish National Tourist Organization, I know other members, they are retired or are pensioners, so we meet and travel together. Each of us has something in his backpack, and we camp and drink beer' (M, PL).

\section{Specific Cultural Differences between the Eight Countries}

We found some particularities about alcohol consumption amongst the different countries. Narratives about alcohol drinking are situated within a changing life course context. The most marked events that changed alcohol consumption are related to social and health aspects, which have a different meaning for each country. Some of the reports from the eight countries illustrate these differences.

In Denmark there is a difference in the perception and the use of different alcoholic beverages. As mentioned before, older people in Denmark report drinking wine with their dinner and stress that moderate consumption has some health benefits. This attitude is probably due to the fact that there has been some focus in the media on that drinking one glass of wine every day can prevent different kinds of illnesses. The older people indicate that they believe that wine is healthy because it reduces the risk of stress and coronary heart disease: 'My husband and I, we like to share a bottle of red wine. We have it for two days, then. It is supposed to be healthy. But we don't get it every day, if that is what you mean' (F, DK).

Polish narratives become special due to the food habits of older people. Poles traditionally eat 'heavy' meals especially in winter and they consume alcoholic beverages to improve their digestion in this season. Some individuals reported a change on the type of alcoholic beverage consumed when comparing to the past in some countries. Polish seniors mentioned they changed 
from drinking vodka to wine over the last years, mirroring the trends in younger generations of Poles.

Generational differences in alcohol consumption and the changing role of alcohol over their life course were often referred by the UK respondents, either positively or negatively. Older people in the UK have seen alcohol consumption increase in the last 10-15 years, which, for many, is alien to former consumption habits. Retirement meant an increased flexibility in routine and socializing which was perceived to increase opportunities to drink, although, sometimes, it also limited financial resources available to spend on alcohol. Generational differences of consumption are therefore central to many accounts as well as the negative aspects of alcohol in the UK. Alcohol consumption has different meanings for individuals of different partnership status and living circumstance, which are vulnerable to change in later life.

Sweden, historically, has not been a country of wine drinkers, although this is now changing in some sections of the population. When talking of alternative alcoholic beverages (to beer) wine is the most common one. The use of wine instead of beer and strong liquor and the association between 'drink not drive' is however not specific for older people-it is a pan-Swedish feature. When describing a dinner party and the use of alcohol in relation to that a lot of the informants in Sweden mention the necessity to take alternative transport home. They talked about needing to order a taxi, or to go by bicycle or bus. However these participants rarely mentioned strong alcohol, although some, especially the men, described the consumption of spirits as something they did when they were younger. Another issue, perhaps typically Swedish, is the common use of light beer. Light beer is cheap, it is available in every store and it is often offered at lunch restaurants as a part of the meal (in these restaurants it is common to offer as a meal: a beverage, salads, bread and coffee). The beverages available are often: light beer, sparkling water, milk or juice.

Portuguese elderly people can be included in the group of countries that do not see drinking as a problem for them, although they viewed alcoholism and social problems of heavy drinking of great importance. As for Italy and Spain, wine and beer are a traditional part of the culture from early years. This brought a particularity to alcohol consumption narratives from the older people, especially men, because the narratives regarding alcohol consumption were casual during the interviews or mentioned only when they were no longer able to drink because of health constraints. But in general, they assumed that drinking a glass of wine is a normal behavior (is part of the meal), and thus alcoholic consumption was likely to be underreported in some cases.

The Spanish narratives showed that alcoholic beverage consumption seems to decrease as age increases, because these people perceive that the regular consumption can be bad for their health, and therefore in some cases, is incompatible with medication. This situation is also common in Portugal. In some reports (IAS, 1999) we found that alcohol consumption usually declines 
with age and the proportion of non-drinkers increases. In Spain, we found that the diagnosis of some disease or the recommendations to prevent any abnormal situation regarding increased blood sugar or blood cholesterol can modify food habits in general, including alcoholic beverages. Some of these older people who used to consume alcoholic beverages regularly had stopped drinking them.

\section{Discussion and Reflection}

This is a first approach to studying alcohol consumption in eight European countries and will help us understand older people's current attitudes and beliefs towards alcohol use. However, we are mindful that, as with most pioneering work, the study has weaknesses as well as strengths.

The general overview of the attitudes regarding alcohol consumption in these eight European countries was a result of the qualitative analysis made by each partner. The strengths lie in its richness of data, collected contemporaneously with identical interview schedules and analyzed with the same code trees generated and agreed by the partners, in a 'Euro-friendly' software program across the eight countries. We identified the value of a grounded theory approach to analysis, whereby concepts evolved from the data rather than from hypotheses. So, although alcohol consumption was not an explicit question in the schedule, most respondents reported its consumption for efficacious health and social reasons. Even those who were abstainers, either lifelong or more recently as a result of deterioration in health, discussed alcohol and its effects on them. Very few did not mention it at all. We consider therefore that this is a largely under-researched field in the study of older people.

Nevertheless, despite rigorous attempts to standardize analytical procedures through the use of the European based software program MAXqda (2001), it is possible that some of the data may have been 'lost in translation' once the findings were presented in English. Also, for some countries, any bias could be attributed to the sample itself. This is because the interviews were carried out in only one or two geographical areas from the countries usually in the environs of the universities involved with the project. For example, the German report notes that their sample belonged to the south where wine is more commonly consumed than beer; in the north of the country the opposite would apply. In Portugal, the data was only collected in the north of the country, which has a large rural population. Some of the oldest participants were illiterate and alternative ways of filling the food shopping and consumption diaries had to be found (telephoning or revisiting the participants). On the other hand, the UK sample was derived from the southeast of England, which is considered an affluent region. Nevertheless, there are pockets of deprivation and the researchers sought out people from different socio-economic groups within the region.

A major limitation is that measuring levels of consumption, which relies 
on self-reporting, is likely to underestimate actual units consumed (Schonfeld and Dupree, 1994). Alcohol consumption assessment is considered a very sensitive issue because it has a highly symbolic value, under the influences of cultural and social norms. The method used in to assess alcohol consumption attitudes and beliefs in these samples were face-to-face interviews, using a semi-structured protocol, and one of the disadvantages of this method was that they did not always provide enough information regarding this issue (ILSI Europe Alcohol Task Force, 1999).

\section{Conclusions}

This article is based on the narratives of alcohol consumption obtained from a sample of 644 old people from 8 European countries, living in the community in 2004. It is a first approach to a subject of current and increasing interest and which deserves further investigation. Although there were selfreported 'heavy' drinkers in the sample, our primary focus was on alcohol and its meaning for commensality and perceived quality of life.

We found cultural differences, principally between northern and southern Europe and their attitude to alcohol and alcohol consumption. We found that in societies with an ambivalent, morally charged relationship with alcohol (such as the Nordic countries), 'celebration' is used as an excuse for drinking; whereas in societies in which alcohol is a morally neutral element of life (such as Mediterranean countries), alcohol is strongly associated with celebration but not seen as a justification for every drinking occasion. In the Nordic countries, the participants were far more defensive about their alcohol consumption. They were anxious that they were not considered 'alcoholic' if they 'indulged' in lone drinking. They were also more concerned with the alcohol related behaviours of younger cohorts. Participants from the Mediterranean countries were far more likely to view alcohol consumption, especially wine, as a customary accompaniment to meals and social interaction. However, even these respondents assured us they 'drank only little', or 'have just a taste'.

There were substantial gender differences in narratives of alcohol, but less marked differences between the countries. In all cultures, the women tended to drink less than the men, and in later life, tended to have reduced their consumption more significantly than men. Both men and women were likely to reduce drastically, or cease drinking alcohol in the presence of very poor health, but women would give up sooner. Other life transitions such as widowhood also showed different patterns. Widows, for example, reported a reduction in alcohol consumption as a result of living alone: 'I don't drink on my own now', since they do not have their spouse to 'share' with them especially at mealtimes. They were also extremely unlikely to go to a pub, tavern or restaurant on their own, but will on occasion go with other women. Men who were widowed on the other hand, were more likely to go out and eat 
alone and have a drink, "I come, I say 'hello', eat and have my glass of beer or my glass of wine, and then I leave again". In all cultures, the men, regardless of marital status, found the company of other men enhanced by the presence of alcohol.

However, for the vast majority of the participants, male or female, alcohol provided the 'social glue' of an ideal or proper meal, which was one shared with family and friends, whether a special occasion or routinely. The meal events or occasions for including an alcoholic beverage have changed little over time. However, some participants mentioned they included beer or other alcoholic beverages with other food events, such as snacks during the afternoon, now that they were retired. Nevertheless, drinking wine during the main meal has historically been the most common alcoholic beverage among Mediterranean countries.

On the other hand, older people from northern European countries started to include wine more frequently than when they were younger, and the consumption of spirits less than in former years. These occasions are in many cases linked to commensality, social determinants and culture. However, the choice of the type of drink, how much to drink, with whom to drink in what situation, are increasingly being shaped by global factors such as easier access to a wider variety of products, the media and the influence of younger generations including adult children.

We have information on how alcohol consumption has altered over the lifecourse and what might be the predisposing and precipitating social and health factors in change, but we were not able, from our data, to make cohort comparisons, that is comparing older with younger generations. We consider we have offered valuable insights into the significance of alcohol in the social networks and behaviours of a large number of elderly people. It would be very interesting to replicate a project such as ours, when current young and middle-aged cohorts in Nordic countries reach old age, having become more accustomed to Mediterranean licensing hours and attitudes to alcohol. Will globalization reduce the north/south divide we have identified? We can only speculate that, as the WHO (2002) reported, deeprooted cultural framing and meaning of drinking behaviours are resistant to change.

\section{Biographical Notes}

Corresponding authors: Maria Daniel Vaz de Almeida, Faculdade de Ciências da Nutrição e Alimentação, Universidade do Porto, R. Dr. Roberto Frias 4200-465 Porto. Portugal. E-mail: mdvalmeida@fcna.up.pt ; Kate Davidson, University of Surrey, Guildford, Surrey, GU2 7XH, UK. E-mail: K.Davidson@surrey.ac.uk.

Maria Daniel Vaz de Almeida PhD belongs to the Faculty of Nutrition and Food Sciences (FCNAUP), where she is the Head of the Faculty and Professor of Community Nutrition. She has been involved in several European research projects in nutrition and public health.

Kate Davidson PhD is a co-director of the Centre for Research on Ageing and Gender (CRAG) at the 
Sociology Department at the University of Surrey. CRAG brings together social scientific expertise to conduct policy relevant research on gender and ageing. The principal aim is to advance understanding of how gender influences the experience of ageing, and how ageing influences gender roles and relationships across the life course.

\section{Acknowledgements}

The data used in this article belong to the European Research Project Food in Later Life. Choosing foods, eating meals: sustaining independence and quality of life (2004-2005) directed by Dr. Margaret Lumbers and Dr. Monique Raats from the University of Surrey (United Kingdom) and financed by the European Commission (QLK1CT200202447).

\section{Notes}

1. Faculdade de Ciências da Nutrição e Alimentação da Universidade do Porto, Portugal.

2. University of Surrey, UK.

3. Grup d'Estudis Alimentaris, University of Barcelona, Spain.

4. MAPP, The Aarhus School of Business, Denmark.

5. Department of Human Nutrition, Warsaw Agriculture University, Poland.

6. Istituto Nazionale di Ricerca per gli Alimenti e la Nutrizione (INRAN), Italy.

7. Department of Public Health and Caring Sciences, Uppsala University, Sweden.

8. Federal Research Centre for Nutrition (FRCN) (Bundesforschungsanstalt für Ernaehrung), Germany.

\section{References}

Chin, A.H., O' Connell, H. and Cunningham, C. (2004). Alcohol use disorders and the elderly. Geriatric Medicine, 34(6), 44-49.

Clough R., Hart R., Nugent M., Fox, D. and Watkins C. (2004). Older people and alcohol. A summary and recommendations arising from research in Ayrshire and Arran. Lancashire: Third Sector First.

Djousse, L. R. E., Beiser, A., Scaramucci, A., D’Agostino, R. B. and Wolf, P. A. (2002). Alcohol consumption and risk of ischaemic stroke. Stroke, 33(4), 907-12.

Hepple, J. (1996). Alcohol and older people. Elderly Care, 8(6), 34-35.

Institute of Alcohol Studies (IAS). (1999). Spring. Alcohol and the elderly, IAS Fact Sheet. St Ives, Cambridge UK: IAS.

Institute of Alcohol Studies (IAS). (2005). Winter. Alcohol consumption and harm in the $U K$ and EU, IAS Fact Sheet. St Ives, Cambridge UK: IAS.

International Life Science Institute Europe Alcohol Task Force. (1999). September. Overview of the health issues related to alcohol consumption, Executive summary of the book 'Health issues related to alcohol consumption'. 2nd edition Brussels: Blackwell Science Ltd.

Lofland, J. and Lofland, L. (1995). Analysing social settings: A guide to qualitative observation and analysis. Belmont, CA: Wadsworth.

MAXqda (2001). Software for qualitative data analysis. Berlin: VERBI Gmbh.

Schonfeld, L. and Dupree, L. W. (1994) Alcohol abuse among older adults. Reviews in Clinical Gerontology, 4 (3), 217-225. 
O'Connell, H., Chin, A.-V., Cunningham, C. and Lawlor, B. (2003). Alcohol use disorders in elderly people - redefining an age old problem in old age. British Medical Journal, 327, 664-667.

Silverman, D. (1993). Interpreting qualitative data: Methods for analysing talk, text and interaction. London: Sage.

Social Issues Research Centre. (1998). Social and cultural aspects of drinking. A report to the Amsterdam group. Oxford: Social Issues Research Centre.

Strauss, A. and Corbin, J. (1990). Basics of qualitative research, Grounded theory procedures and techniques. Newbery park, CA: Sage.

Thun, M., Peto, R. and Lopez, A. (1997). Alcohol consumption and mortality among middle aged and elderly US adults. New England Journal of Medicine, 337(24), 170514.

Truelsen, T., Thudium, D. and Gronbaek, M. (2002). Amount and type of alcohol and risk of dementia: the Copenhagen city heart study. Neurology, 59(9), 1313-19.

Vestal, R., McGuire, E., Tobin, J., Andres, R., Norris, A. and Mezey, E. (1977). Ageing and ethanol metabolism. Clinical Pharmacological Therapy, 21, 343-54.

World Health Organization (WHO). (2002). Management of substance dependence noncommunicable diseases. A Summary of Alcohol in Developing Societies: A Public Health Approach: Geneva: WHO.

World Health Organization (WHO), Department of Mental Health and Substance Abuse. (2004). Global status report on alcohol 2004. Geneva: WHO.

Wilsnack, S. C. and Wilsnack, R. W. (2002). International gender and alcohol research: recent findings and future directions. Alcohol Research and Health, 4(26), 245-250. 